\title{
ACTIVIDAD FÍSICA, ß ENDORFINAS Y PSICOTERAPIA SINÉRGICO FUNCIONAL (TSF): JUDO-TERAPIA VERBAL-HIPNOSIS
}

\author{
Dutari, Ildefonso \\ Hospital Sanatorio Las Cumbres, Universidad de Panamá \\ Panamá, Panamá \\ ildefonso.dutari@meduca.gob.pa \\ clement551@hotmail.com \\ Malgrat, Carlos (q.e.p.d.) \\ Hospital Sanatorio Las Cumbres, Universidad de Panamá \\ Panamá, Panamá

\section{Resumen}

El objetivo es demostrar la relación entre la actividad física, la secreción de ßendorfinas y su implicación en la Psicoterapia Sinérgica Funcional (PSF): Judo-Terapia Verbal-Hipnosis. Las tres técnicas producen mejora del consultante, a través de la emisión de ßendorfinas, que juegan un papel integral hormonal-metabólico a la respuesta al ejercicio. En la PSF: Judo-Terapia Verbal-Hipnosis, se produce una sinergia funcional de las tres técnicas, las que al actuar independientemente se conjugan en una sola. El judo es el deporte que debido a la alta producción de ßendorfinas promueve relajamiento, seguridad y bienestar al paciente. Su rol en la terapia es el más efectivo. La terapias verbales son de diferentes tipos en el modelo de mejora. La hipnosis coadyuva a través de las sugerencias produciendo relajamiento del individuo. El estudio clínico consistió en una muestra de sujetos: 723 en total, 401 (55.46\%) hombres y 322 (46.54\%) mujeres en los casos en que se completaba la mayor parte o parte del tratamiento. Se han logrado resultados óptimos utilizando esta terapia, tanto en casos como la fobia social o en subtipos de psicosis. Se ha registrado que el paciente tiene un tope en su recuperación, pero no involuciona a estadios tempranos de la alteración. Se está elaborando un modelo neurofisiológico de la PSF, que incluye las ßendorfinas.

Palabras claves: Actividad física, Betaendorfinas, Sinergia Funcional, Psicoterapia Sinérgico Funcional, modelo neurofisiológico. 


\section{Abstract}

The objective is to demonstrate the relationship between physical activity, the secretion of beta endorphins and their involvement in Functional Synergistic Psychotherapy (FSP): JudoVerbal Therapy-Hypnosis. The three techniques produce improvement of the consultant, through the emission of ßendorphins, which play an integral hormonal-metabolic role to the response to exercise. In the FSP: Judo-Verbal Therapy-Hypnosis, there is a functional synergy of the three techniques, which when acting independently are combined in a single one. Judo is the sport that due to the high production of ßendorfins promotes relaxation, safety and well-being to the patient. It's role in therapy is the most effective. Verbal therapies are of different types in the improvement model. Hypnosis contributes through suggestions producing relaxation of the individual. The trial consisted of a sample of subjects: 723 in total, 401 (55.46\%) male and 322 (46.54\%) women in cases where most of it was completed most or part of the treatment. Optimal results have been achieved using this therapy, both in cases such as social phobia or in subtypes of psychosis. It has been recorded that the patient has a top on his recovery, but does not regress to early stages of the alteration. A neurophysiological model of FSP, including ßendorfins, is being developed.

Keywords: Physical activity, Betaendorphins, Functional Synergy, Functional Synergistic Psychotherapy, neurophysiological model.

\section{INTRODUCCIÓN}

El judo como terapia ha sido investigado y aplicado por otros psicoterapeutas como Callan (2018) [1], González Pando [2], Scuderi (1995) [3], Martínez Suárez, Gleser J., Brown P. (1988) [4], con buenos resultados. El judo proporciona seguridad (Brousse \& Matsumoto (1999) [5], que tiene gran implicación y nuevas posibilidades en la actividad mental y en los estudios emocionales. Se considera que esta actividad psicomotora al producir $\beta$ endorfinas produce relajamiento y bienestar al paciente. Su rol en la terapia es muy efectivo. Esto es confirmado por Dutari (1955) [6] [7] en otros estudios.

La terapia verbal directiva es relevante en el cambio de la estructura mental, como forma de orientar y canalizar al paciente hacia la confrontación y mejora de su problema. Se han utilizado la terapia directiva Malgrat \& Dutari (1986) [8] los principios de la neurolingüística y otras psicoterapias: dialéctica Linehan (2001) [9] y cognitivo conductual. La terapia cognitivo-conductual (Ellis, Caballo y Lega) [10] asume que los patrones de pensamiento 
erróneos causan un comportamiento desadaptativo.

La hipnosis contribuye en la terapia en dos formas: haciendo las sugerencias de acuerdo al caso particular y para producir relajamiento del individuo. Se han utilizado la hipnosis tradicional y la ericksoniana. La hipnosis tradicional se basa en la sugestión, pero la hipnosis ericksoniana se basa en estados de relajación más o menos profundos y en la participación activa del paciente.

Un hecho que se ha podido observar, pero no se ha podido elaborar el mecanismo de explicación es que una vez el individuo llega a un estado tope de mejora, pero no involuciona. Se trabaja en un modelo neurofisiológico de la terapia.

\section{METODOLOGÍA}

Sujetos: 723 en total, 401 (55.46\%) hombres y 322 (46.54\%) mujeres. Los pacientes eran los ingresados de los Hospital Psiquiátrico de Las Cumbres. Eran sobre todo niños, adolescentes y adultos jóvenes que puedan practicar judo, o sea, sin problemas físicos o de tipo cardiovascular.

Materiales: Los materiales serán las pruebas psicométricas, se necesitará material de oficina (hojas blancas, carpetas, etc.), fotocopias de las pruebas, material para docencia con los judocas, con los esquizofrénicos, gimnasio.

Las pruebas psicológicas fueron: Rorschach, PMK (Psicodiagnóstico Miokinético) MMPI, Bernreuter.

Recursos: Existían tres salas de reuniones para grupos de investigación, una sala de rapport y una sala para administración de pruebas. Además, se requiere de compra VHS, equipo de grabación, para videos (para el análisis de las sesiones).

Ejecución de la Terapia: La terapia se hizo mayormente en el Hospital Sanatorio Las Cumbres (ya cerrado), en las consultas privadas de los psicólogos e investigadores Dr. Carlos Malgrat e Ildefonso Dutari R. Ha durado más de 30 años en lograr esa anamnesis. 


\section{RESULTADOS}

Fig. 1

Cantidad de Consultantes Mejorados

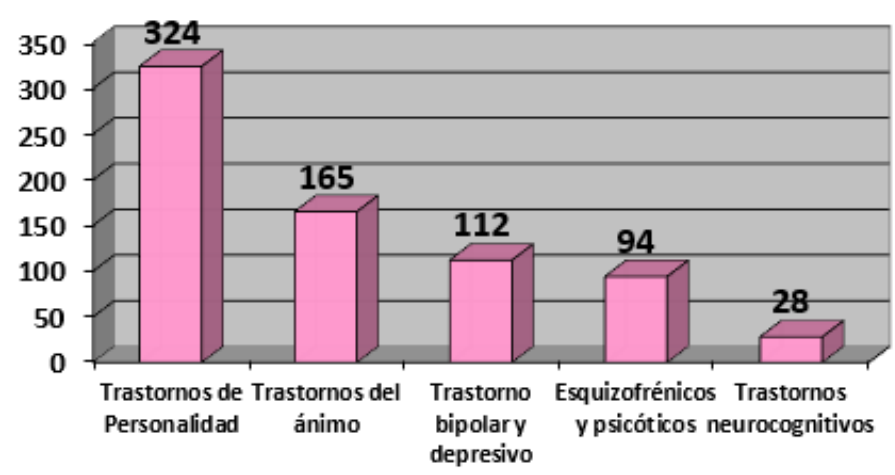

Alteraciones Psicológicas

$\square$ Cantidad de Pacientes

Fig. 2

Trastornos Psicóticos

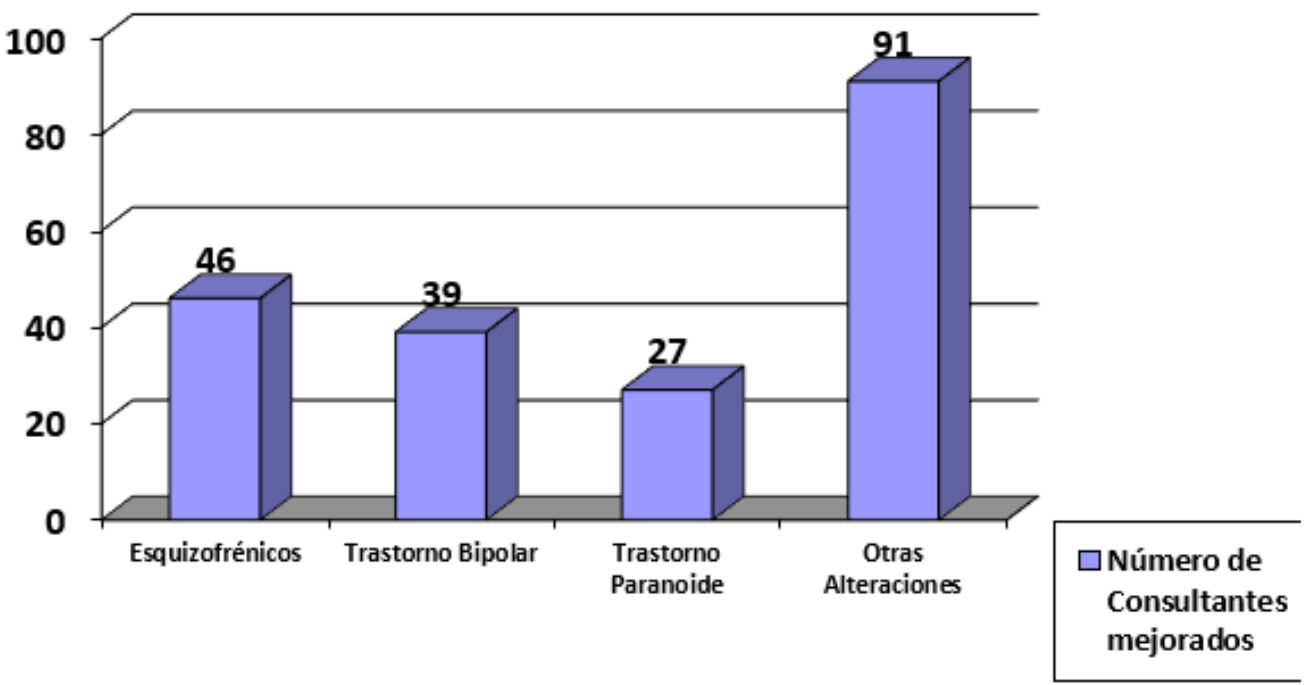

\section{CONCLUSIONES}

La combinación de las tres técnicas: judo, terapia verbal directiva (u otras) e hipnosis actúan como una sinergia funcional y los resultados son mejores que si actúan independientemente.Las tres convergen en el sistema funcional complejo alterado del cerebro, transformando el sistema neuronal y adaptándolo inter e intrapsíquicamente. 
Se han logrado resultados óptimos utilizando esta terapia, tanto en casos sencillos de inadaptación social como en casos de psicosis incipientes: esquizofrenia simple, trastorno bipolar. Existe un hecho registrado: el paciente tiene un tope en su recuperación, pero no involuciona a estadios tempranos de la alteración. Es un nuevo modelo. Se está elaborando un modelo neurofisiológico de la terapia.

\section{Referencias}

[1] Callan, M. \& Bradic, S., (11 Jun 2018) Applicable research in judo: Proceedings book: 5th European Science and Judo Research Symposium. Corak, S., Sertic, H. \& Segedi, I. (eds.).pp. 23-27,Porec, Croatia: University of Zagreb.

[2] González Pando, D., Beltrán García, P. y Martínez Suárez, P.C. (2016). Explorando el potencial de aprendizaje en personas con esquizofrenia en diferentes condiciones psicopatológicas. Clínica y Salud, 27(2), 81-88.

[3] Scuderi, A. J. (1995) The psychospirituality of systems centered therapy: mind-soul-body wellnes, a holistic approach.Judo informationsite copyright.

[4] Gleser J., Brown P.(1988) Judo principles and practices: applications to conflict-solving strategies in psychotherapy. Am J.Psychother.Jul;42(3):437-47

[5] Brousse, M. \& Matsumoto, D. (1999).Judo, a sport and a way of life, Seoul: International Judo Federation.

[6] Dutari, I. (August, 27-30 1995) Functional synergic psychotherapy: judo-verbal therapy-hypnosis. European Journal of Psychological Assesment. 3rd European Conference of Psychological Assesment, v. 11, sup. 1,p. 103, Trier, Germany: Hogrefe \& Huber Publishers.

[7] Dutari-Ruíz, I., Caso clínico de aplicación: El estudiante problemático. Delegación Norte del Colegio Oficial de Psicólogos, 29 mar. 1995, Oviedo, España) (Oral)

[8] Malgrat, C. \& Dutari, I. (1986) La psicoterapia sinérgico funcional: primeros hallazgos. Revista del hospital sanatorio Las Cumbres. Panamá.

[9] Ellis, A., Caballo, V. E., Lega, L. I. (2009) Teoría y práctica de la terapia racional emotiva-conductual. Madrid: Siglo XXI de España.

[10] Linehan, M. (2003) Manual de tratamiento de los trastornos de la personalidad. Madrid: Paidós Ibérica.

[11] Erickson, M. H. (2001) Clínica e hipnosis. Madrid: Paidós: Ibérica.

\section{Autorización y Licencia CC}

Los autores autorizan a APANAC XVIII a publicar el artículo en las actas de la conferencia en Acceso Abierto (Open Access) en diversos formatos digitales (PDF, HTML, EPUB) e integrarlos en diversas plataformas online como repositorios y bases de datos bajo la licencia CC:

Attribution-NonCommercial-ShareAlike 4.0 International (CC BY-NC-SA 4.0) https://creativecommons.ora/ licenses/by-nc-sa/4.0/.

Ni APANAC XVIII ni los editores son responsables ni del contenido ni de las implicaciones de lo expresado en el artículo. 